\title{
Skin rash associated with intravitreal bevacizumab in a patient with macular choroidal neovascularization
}

\author{
loannis D Ladas \\ Marilita M Moschos \\ Thanos D Papakostas \\ Athanasios I Kotsolis \\ Ilias Georgalas \\ Michail Apostolopoulos \\ Department of Ophthalmology, \\ "G. Gennimatas" Hospital of Athens, \\ University of Athens, Athens, Greece
}

Correspondence: Marilita M Moschos

I44 Kountouriotou Street, 18535

Piraeus, Greece

Tel +306944887319

Fax +30 21 0412 2139

Email moschosmarilita@yahoo.fr
Purpose: The purpose of this observational case report is to describe a case of skin rash after intravitreal use of bevacizumab.

Methods: A 50-year-old man with choroidal neovascularization in the right eye due to age-related macular degeneration was treated with three intravitreal injections of bevacizumab.

Results: Twelve days after the first injection, the patient developed a maculopapular rash on his forehead and on both temporal regions around his eyes. The rash disappeared eight days after treatment with topical corticosteroids. A skin rash with the same distribution reappeared 14 days after the second and 10 days after the third injection. Similarly, it disappeared five and seven days after the use of the same treatment. The follow-up period was 15 months after the third injection. During the follow-up period the rash did not reappear.

Conclusion: This case report may initiate further investigation of similar cases to support this observation, as there are a lack of reports of skin rash after intravitreal administration of bevacizumab.

Keywords: skin rash, bevacizumab, age-related macular degeneration

\section{Case report}

Bevacizumab is a recombinant humanized antibody that binds to all isoforms of vascular endothelial growth factor (VEGF). It is designed for intravenous use in the treatment of metastatic colorectal cancer, ${ }^{1}$ but it is also offered as an off-label intravitreal application for retinal disorders, such as exudative age-related macular degeneration (ARMD). ${ }^{2}$ We report the development of a skin rash in a patient with macular choroidal neovascularization (CNV) due to ARMD, who was treated with repeated intravitreal injections of bevacizumab.

A 50-year-old man presented to us with decrease of vision in the right eye (RE) for the last two months. Fundus examination, fluorescein angiography (FA) and optical coherence tomography (OCT) revealed a parafoveal minimally classic CNV with exudative lesions in the right eye due to ARMD. Visual acuity (VA) was 3/10 RE $\left(-0.5-2.25145^{\circ}\right)$ and $10 / 10$ left eye (LE) $\left(-1.0-1.535^{\circ}\right)$. A treatment with repeated intravitreal injections of bevacizumab was decided and the patient signed an informed consent form for the acceptance of this treatment.

All the intravitreal injections were carried out in the operation theatre. Topical proparacaine hydrochloride $0.5 \%$ was applied to the ocular surface followed by preparation with $5 \%$ povidone iodine. A cotton-tipped applicator soaked in proparacaine hydrochloride was then applied to the injection site $4 \mathrm{~mm}$ posterior to the limbus. The intravitreal dose of bevacizumab (Avastin) was $1.25 \mathrm{mg} / 0.05 \mathrm{ml}$. Bevacizumab was placed in a $0.5 \mathrm{ml}$ syringe with an integrated 31 -gauge needle and $0.2 \mathrm{ml}$ was 
injected into the vitreous. Indirect ophthalmoscopy was used to confirm uneventful intravitreal placement of the suspension.

Twelve days after the first intravitreal injection of bevacizumab (1.25 mg), a maculopapular rash appeared on the forehead (Figure 1), as well as on both temporal regions around the eyes (Figure 2), while he didn't have this specific rash elsewhere on the body. The rash disappeared seven days later after treatment with topical corticosteroids (ointment betamethazone valerate $0.1 \%$ three times a day on the area of skin rash). A skin rash with the same distribution was reappeared 14 and 10 days after the second and the third intravitreal injection of bevacizumab, which also disappeared seven and 10 days after the intravitreal injection.

After the third injection, the VA of the RE improved to $8 / 10$ and the exudative lesions disappeared. The patient was followed-up without any treatment until 15 months after the third injection. The VA remains stable without recurrence of the exudative lesions. In addition, the skin rash did not reappear during the whole follow-up period.
The most common side effects associated with the intravenous use of bevacizumab are hemorrhage, arterial thromboembolism, gastrointestinal perforation, and hypertension. ${ }^{3}$ Gotlib and colleagues ${ }^{4}$ described a rash similar to our patient's after intravenous use of bevacizumab for the treatment of colon cancer. Other dermatologic side effects which have been described after the systemic use of bevacizumab include exfoliative dermatitis ${ }^{5}$ and unspecified rash. ${ }^{6}$ The intravitreal administration of bevacizumab may be followed by side effects including inflammation or uveitis, endophthalmitis, lens injury, cataract progression, subretinal hemorrhage, retinal detachment, blood pressure elevation and retinal pigment epithelium tears. ${ }^{7}$

We believe that the development of our patient's rash was linked to the intravitreal injection of bevacizumab, as it always appeared with the same distribution after every of the three repeated intravitreal injections. All three times, the skin rash appeared 10-14 days after the injection and disappeared 7-10 days later after the use of treatment with topical corticosteroids. Further, the skin rash did not reappear

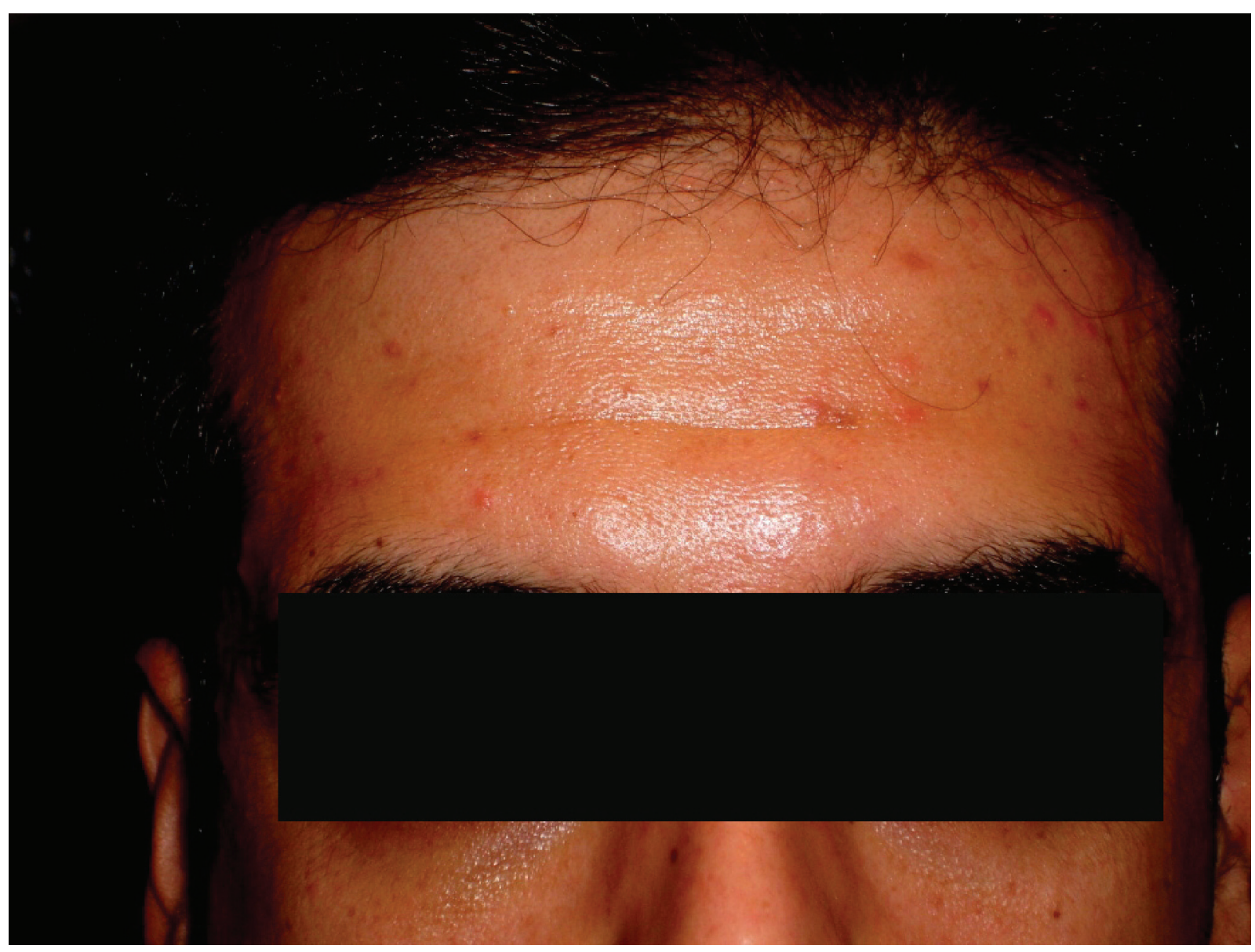

Figure I The maculopapular rash on the patient's forehead. 


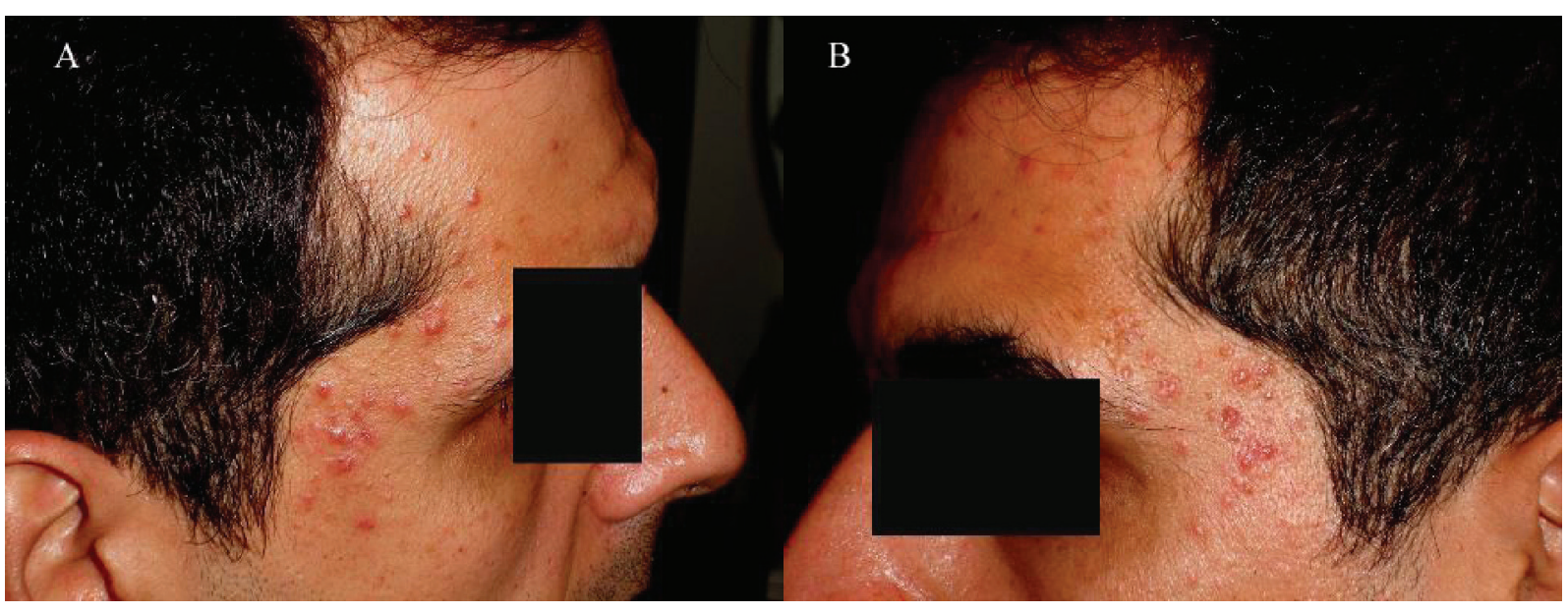

Figure 2 The maculopapular rash on the patient's temporal regions around his eyes: right (A) and left (B).

when the intravitreal injections were interrupted for a period of 15 months after the third injection.

We believe that this rash was not a local reaction to the topically administered antibiotics or the betadine, as it was developed not only around the treated eye but also around the other eye.

To our knowledge, this is the first report of development of a skin rash after intravitreal injection of bevacizumab. Skin rashes have not yet been reported with ranibizumab, the antibody fragment of bevacizumab, which has been approved for the treatment of ARMD. We have also not documented any case of skin rash after intravitreal administration of ranibizumab.

A possible explanation for the development of this adverse event is the functional similarity of VEGF with the epidermal growth factor receptor (EGFR). Skin rashes are a typical side effect of EGFR antagonists, such as the antibody cetuximab. ${ }^{8}$ They usually develop two weeks after the intravenous administration of the drug and affect the face, trunk, and extremities. Furthermore, the blockage of VEGF by other drugs such as BAY 43-9006 results to the development of skin rashes along with its other side effects. ${ }^{9}$

In conclusion, a possible rare side effect that may occur after an intravitreal injection of bevacizumab is the development of a skin rash. This case report may initiate further investigation of similar cases to support this observation, as there are a lack of reports of skin rash after intravitreal administration of bevacizumab.

\section{Disclosure}

The authors report no conflicts of interest in this work.

\section{References}

1. Ferrara N, Hillan KJ, Gerber HP, et al. Discovery and development of bevacizumab, an anti-VEGF antibody for treating cancer. Nat Rev Drug Discov. 2004;3:391-400.

2. Michels S, Rosenfeld PJ, Puliafito CA, et al. Systemic bevacizumab (Avastin) therapy for neo-vascular age related macular degeneration: twelve-week results of an uncontrolled open-label study. Ophthalmology. 2005;112:1035-1074.

3. Hurwitz H, Saini S. Bevacizumab in the treatment of metastatic colorectal cancer: safety profile and management of adverse events. Semin Oncol. 2006;33:S26-S34.

4. Gotlib V, Khaled S, Lapko I, et al. Skin rash secondary to bevacizumab in a patient with advanced colorectal cancer and relation to response. Anticancer Drugs. 2006;17:1227-1229.

5. Motl S. Bevacizumab in combination chemotherapy for colorectal and other cancers. Am J Health Syst Pharm. 2005;62:1241.

6. Gordon MS, Margolin K, Talpaz M, et al. Phase I safety and pharmacokinetic study of recombinant human anti-vascular endothelial growth factor in patients with advanced cancer. J Clin Oncol. 2001;19:843-850.

7. Fung AE, Rosenfeld PJ, Reichel E. The International Intravitreal Bevacizumab Safety Survey: using the internet to assess drug safety worldwide. Br J Ophthalmol. 2006;90:1344-1349.

8. Cunningham D, Humblet Y, Siena S, et al. Cetuximab monotherapy and cetuximab plus irinotecan in irinotecan-refractory metastatic colorectal cancer. N Engl J Med. 2004;351:337-345.

9. Ratain MJ, Eisen T, Stadler WM, et al. Phase II placebo-controlled randomized discontinuation trial of Sorafenib in patients with metastatic renal carcinoma. J Clin Oncol. 2006;24:2505-2512. 
